\title{
Diagnostic des périodes de contrainte hydrique chez des plantes d'aubergine cultivées en serre, au moyen des microvariations des tiges
}

\author{
N Katerji 1, PG Schoch 2, P Rimgoto 2, JC L'Hotel 2 \\ avec la collaboration technique de B Brunel, JL Luciani, B Durand \\ 1 INRA, Station de bioclimatologie, 78850 Thiverval-Grignon; \\ 2 INRA, Station de bioclimatologie, BP 91, 84140 Montfavet, France
}

(Reçu le 25 octobre 1989; accepté le 17 juin 1990)

\begin{abstract}
Résumé - Au cours de deux expériences réalisées en serre sur des plantes d'aubergine soumises à des apports d'eau différents, les auteurs ont suivi la variation des diamètres des tiges et des indicateurs de l'état hydrique de la plante. L'objectif étant de vérifier s'il existe des valeurs seuils de variation de diamètre, révélatrices d'un manque d'eau et susceptibles d'être utilisées par la suite pour un diagnostic de la contrainte hydrique. Les résultats obtenus indiquent d'une part que les valeurs seuils peuvent être déterminées d'une façon quantitative à partir de 2 critères : la contraction maximale journalière $(C M)$ et l'évolution nette du diamètre maximal $(D M)$ - la précision affectant ces critères déterminés à partir de 7 capteurs varie de 30 à $35 \%$-, d'autre part qu'une évolution négative du diamètre maximal peut être considérée comme un critère qualitatif pour diagnostiquer une contrainte hydrique. Enfin, les auteurs suggèrent de retenir le potentiel de base comme référence pour déterminer les valeurs seuils de variation du diamètre, en raison notamment de la sensibilité de cet indicateur de l'état hydrique de la plante à un changement du régime hydrique.
\end{abstract}

transpiration / potentiel hydrique foliaire / résistance stomatique / photosynthèse

Summary - Diagnosis via stem microvariations of water stress periods in greenhouse-grown eggplants. In 2 greenhouse experiments on eggplants submitted to different water supply regimes, variations in stem diameters and in indicators of plant water status were studied. The objective was to determine whether threshold values for stem diameters existed, demonstrating water lack, and which could be used later on in the diagnosis of water stress.

The results obtained indicate that the evolution of stem microvariations is associated with the evolution of several indicators of plant water status such as predawn leaf water potential (figs 2, 3), stomatal resistance, transpiration, leaf water potential and photosynthesis (figs 4,5 ). The authors suggest nevertheless that the predawn leaf water potential be retained as reference in determining threshold values for diameter variation, notably because of the sensitivity of this plant water stress indicator to any change in water regime.

The threshold values can be quantitatively determined from 2 criteria (fig 1): maximum daily contraction (CM) and net evolution of the maximum diameter (DM). The precision affecting these threshold values was studied (tables I and II) and it was noted that it amounted to $\pm 35 \%$ when the $C M$ and DM threshold values were determined with 7 captors. Finally, it is noted that a negative evolution in the maximum diameter can be considered a simple qualitative criterium for the diagnosis of water stress.

transpiration / leaf water potential / stomatal resistance / photosynthesis

\section{INTRODUCTION}

Les premières observations sur l'existence des microvariations des diamètres des organes végétaux au cours de journées ensoleillées remontent à la fin du siècle dernier. Depuis, de nombreux chercheurs ont signalé ce phénomène sur plusieurs espèces végétales, l'ont analysé en relation avec les facteurs du milieu (température de l'air, éclairement, état hydrique du sol, sécheresse atmosphérique), l'ont comparé avec les autres indicateurs de contrainte hydrique chez les plantes (potentiel hydrique foliaire, résistance stomatique, transpiration végétale, etc) et, enfin, l'ont interprété en fonction des connaissances acquises sur les modalités 
du transfert hydrique dans la plante. Une abondante bibliographie a été consacrée à ces sujets, nous ne citerons ici que quelques-uns de ces travaux dans lesquels une bibliographie détaillée peut être consultée (Kozlowski, 1967; Klepper et al, 1971; Molz et Klepper, 1972; Jarvis, 1975; Powell et Thorpe, 1977; Pereira et Kozlowski, 1976; Garnier, 1985; Brough et al, 1986). La plupart de ces travaux ont été réalisés sur des arbres, quelques-uns seulement concernent des plantes herbacées, telles que le soja (So et al, 1979), le maïs (Huguet et al, 1987) et l'aubergine (Schoch et al, 1987).

Des travaux récents ont conduit plusieurs auteurs (Huguet, 1985; Garnier, 1985) à envisager l'utilisation des microvariations des diamètres dans le domaine du pilotage de l'irrigation. En effet, ces mesures peuvent être automatisées et donner lieu à un enregistrement continu en conditions naturelles. La démarche proposée par ces auteurs consiste à : (i) suivre l'évolution des variations de diamètres d'un organe végétal avec un ou plusieurs indicateurs de l'état hydrique de la plante; (ii) déterminer une valeur seuil de ces variations correspondant à l'apparition de la contrainte hydrique; (iii) programmer l'irrigation dès que cette valeur seuil est atteinte. Le bioprogrammateur Pépista, mis au point par Huguet en 1985 , est une réalisation technique fondée sur la démarche décrite précédemment; cet appareillage enregistre les variations de dimensions des organes à partir de 6 capteurs (8 capteurs dans le modèle commercialisé depuis 1988) et déclenche l'ouverture d'une électrovanne d'irrigation quand le seuil critique fixé préalablement est dépassé. Comme on peut le constater, l'utilisation correcte de ce type d'appareillage est conditionnée par la détermination de la valeur seuil correspondant à l'apparition de la contrainte hydrique chez le couvert concerné.

Dans un article précédent (Schoch et al, 1987), la liaison entre la variation du diamètre de la tige et l'état hydrique des plants d'aubergine a été mise en évidence. Dans le présent article, en associant aux observations précédentes des données obtenues dans le cadre d'une expérience inédite réalisée sur le même dispositif expérimental, nous proposons d'aborder l'étape suivante, qui consiste à envisager l'utilisation du signal micrométrique dans le domaine du pilotage de l'irrigation. De façon à caractériser les valeurs seuils de ce signal, nous soulevons trois questions :

- quel est l'indicateur de l'état hydrique de la plante que l'on doit retenir comme référence pour déterminer les valeurs seuils des variations de diamètre ?

- comment déterminer les valeurs seuils: d'une façon qualitative, en associant l'apparition de la contrainte hydrique avec un comportement particulier des variations de diamètre, ou plutôt d'une façon quantitative?

- avec quelle précision détermine-t-on quantitativement les valeurs seuils?

\section{MATÉRIEL ET MÉTHODES}

Les résultats présentés dans cette étude ont été obtenus au centre INRA à Avignon, au cours de deux expériences sur des aubergines (Solanum melongena L) :

- la première a eu lieu pendant 4 journées consécutives, du 11 au 14 juin 1985, sur des plantes semées en avril;

- la deuxième a eu lieu pendant 7 journées consécutives, du 16 au 23 septembre 1985, sur des plantes semées en juillet.

La variété étudiée au cours des expériences (Ronde de Valence) est un cultivar à enracinement superficiel et à forte transpiration en condition de faible évaporation potentielle (Daunay et al, 1986). Les plantes étaient cultivées dans des pots (1 plante par pot) de 7,5 remplis de $8 \mathrm{~kg}$ de terre d'alluvion limonoargileuse. La densité des pots est de 8 au $\mathrm{m}^{2}$ dans un tunnel plastique de $8 \mathrm{~m}$ de large et $20 \mathrm{~m}$ de long. Elles étaient encadrées par des lignes de bordure, et une protection en plastique empêchait l'évaporation directe de la terre.

Les conditions climatiques et le protocole expérimental étaient cependant différents entre les 2 expériences.

\section{Conditions expérimentales de la première expérience (11-14 juin)}

Cette expérience a été réalisée sur des plantes au stade de floraison. Les conditions climatiques au cours des 4 journées étudiées étaient relativement stables. Les températures de l'air mesurées dans le tunnel oscillaient régulièrement entre 13 et $26^{\circ} \mathrm{C}$ en moyenne et l'évapotranspiration potentielle, estimée par la formule de Penman à partir des données recueillies à l'extérieur (Parc météorologique de la station de bioclimatologie d'Avignon), était de l'ordre de 5 $\mathrm{mm} / \mathrm{j}$ en moyenne.

Deux traitements hydriques ont été appliqués aux plantes :

- pendant la période pré-floraison et jusqu'au 10 juin (jour $\mathrm{J}-1$ ), l'humidité du sol est maintenue à la capacité au champ, en effectuant un arrosage quotidien pour compenser la transpiration; 
- l'arrosage est diminué de moitié à partir du 11 juin au soir (jour J), puis arrêté complètement à partir du soir du 12 juin (jour $\mathrm{J}+1$ ).

Des mesures biologiques horaires ont été réalisées au cours des 4 journées étudiées (du jour $\mathrm{J}$ au jour $\mathrm{J}+$ 3) à partir de 5 h 30 (heure légale) jusqu'à 21 h 30 sans interruption, soit 17 mesures par jour.

La résistance stomatique horaire était mesurée sur les faces supérieures et inférieures des feuilles adultes bien dégagées du sommet de la plante, au moyen d'un poromètre à diffusion (MK, II Delta-T Devices, Cambridge). Chaque mesure horaire correspond à la moyenne de 20 mesures (10 sur chaque face). Au même moment, des mesures de l'assimilation nette (10 mesures chaque heure) ont été effectuées sur des feuilles analogues à l'aide de l'appareil LI-6000 de LICOR.

Le potentiel foliaire était mesuré sur des feuilles du sommet de la plante au moyen d'une chambre à pression type Scholander (Scholander et al, 1965). Chaque mesure horaire du potentiel foliaire correspond à la moyenne de 7 feuilles.

Au cours des 4 journées étudiées, les microvariations des diamètres de tige en dessous du premier bouquet ont été mesurées sur 7 plantes ayant des diamètres très voisins, à l'aide de capteurs de déplacement linéaire (Huguet, 1985). Les mesures ont été recueillies en temps réel par un microcalculateur à mémoire permanente (Microcalculateur Husky) doté d'un convertisseur analogique digital pour 8 voies.

La transpiration végétale horaire de 2 plantes a été obtenue en plaçant 2 pots sur une balance enregistreuse au milieu du tunnel plastique. L'observation de fuite d'eau à la suite de l'arrosage provoque une incertitude sur les valeurs mesurées de la transpiration, particulièrement au cours des jours $\mathrm{J}$ et $\mathrm{J}+1$.

\section{Conditions expérimentales de la deuxième expérience (16-23 septembre)}

Cette expérience a été réalisée sur des plantes au stade du premier fruit noué. Les conditions climatiques pendant les 7 journées étudiées étaient stables. Les températures de l'air oscillaient entre 13 et $28^{\circ} \mathrm{C}$, donc de manière assez voisine de celles observées au cours de la 1re expérience; en revanche, l'évapotranspiration potentielle était nettement inférieure $(3,5 \mathrm{~mm} /$ j). Cette différence est directement liée au rayonnement global, plus faible $\left(17 \mathrm{MJ} / \mathrm{m}^{2}\right.$ par j) au cours de l'expérience en septembre, la durée du jour étant plus courte, qu'en juin (24 MJ/m² par j).

Le traitement hydrique appliqué aux plantes était le suivant : jusqu'au 16 septembre, début de la période de mesure, les plantes étaient arrosées quotidiennement pour compenser la transpiration. À partir du 16 septembre, elles ont été divisées en 2 lots et 2 traitements hydriques ont alors été appliqués :

- lot irrigué (témoin) : comme pour la période précédant la nouaison, les plantes étaient arrosées pour compenser la transpiration;
- lot sec : l'arrosage fut arrêté à partir du 16 septembre, jour que nous désignons par J', et remis le 23 septembre le soir $\left(\mathrm{J}^{\prime}+6\right)$.

L'objectif ici était de vérifier par rapport à un témoin l'ensemble des observations recueillies au cours de la $1^{\text {re }}$ expérience.

Nous avons vérifié que les plantes des 2 lots présentaient, le 16 septembre, la même surface foliaire (2 $680 \pm 250 \mathrm{~cm}^{2} /$ plante pour le lot irrigué et $2550 \pm$ 190 pour le lot sec) et la même croissance $(26,5 \pm 3$ g/plante pour le lot irrigué et $25,3 \pm 1,8 \mathrm{~g} /$ plante pour le lot sec).

Des mesures ponctuelles ont été réalisées simultanément sur des plantes des lots sec et irrigué au cours des 7 journées étudiées (du jour J' au jour J' + 6) :

- le potentiel hydrique foliaire était déterminé à des intervalles réguliers, à partir de $8 \mathrm{~h}$ et jusqu'à $19 \mathrm{~h}$, sur des feuilles du sommet de la plante, d'une façon identique à celle décrite au cours de l'expérience du mois de juin et avec le même échantillonnage;

- la résistance stomatique était déterminée sur la face supérieure des feuilles du sommet de la plante à partir de $10 \mathrm{~h}$, et jusqu'à $19 \mathrm{~h}$, heures légales, en même temps que le potentiel foliaire; chaque mesure horaire de résistance stomatique correspondait à la moyenne de 7 mesures réalisées sur chaque lot, à l'aide du même appareillage que celui du mois de juin.

Les microvariations des diamètres des tiges en dessous du $1^{\text {er }}$ bouquet ont été mesurées en continu sur 3 plantes du lot sec et 3 plantes du lot irrigué à l'aide de capteurs de déplacement. Par la suite, nous avons constaté que 2 capteurs seulement de chaque lot ont donné des mesures exploitables de microvariation de diamètres, probablement à cause d'un mauvais contact entre le capteur et la tige mesurée.

\section{Traitement statistique des données}

Les valeurs moyennes horaires des paramètres biologiques (résistance stomatique, potentiel hydrique foliaire et assimilation nette) ainsi que les écarts type ont été calculés heure par heure. La mise en évidence de différences significatives dans l'évolution des paramètres étudiés au cours de l'expérience (fig 4) et la différenciation entre le comportement des plantes appartenant aux lots sec et irrigué (fig 5) ont été déterminées sur la base des données moyennes horaires, et leur intervalle de confiance calculé au seuil de $5 \%$.

\section{RÉSULTATS}

\section{Allure générale des variations du diamètre des tiges}

La figure 1 donne, à titre d'exemple, l'évolution du jour $\mathrm{J}$ à $\mathrm{J}+2$ (11-13 juin) du diamètre de la 


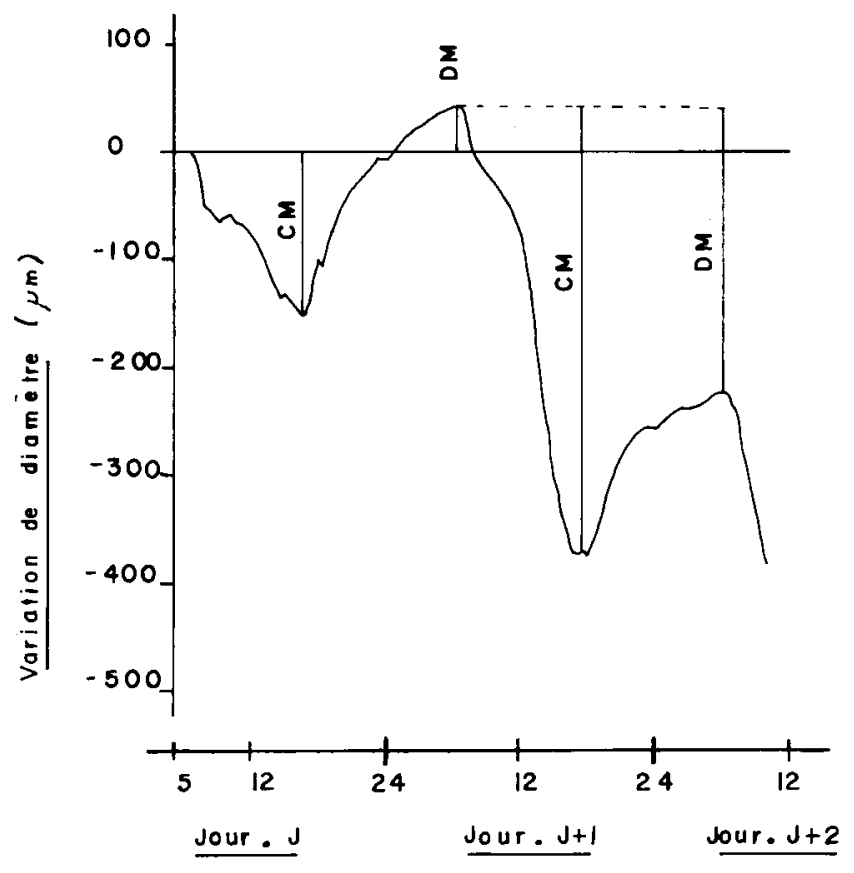

Fig 1. Évolution du jour $\mathrm{J}$ à $\mathrm{J}+2$ du diamètre de tige observé sur un plant d'aubergine (sont signalés sur cette figure les deux critères retenus pour caractériser la journée : $C M=$ contraction maximale, $D M=$ évolution nette du diamètre maximal).

tige d'une plante d'aubergine cultivée dans un sol en phase de dessèchement. On constate que la tige se contracte à partir de 6 ou $7 \mathrm{~h}$ du matin (soit 1 à $2 \mathrm{~h}$ après le lever du soleil) jusque vers 14 ou $15 \mathrm{~h}$ (contraction maximale), puis se dilate pour atteindre un diamètre maximal vers $5 \mathrm{~h}$ du matin. Pour caractériser une journée, 2 critères peuvent être retenus (Kozlowski, 1967) :

- La contraction maximale journalière (CM), qui est la différence entre le diamètre maximal en début de journée avant le début de la contraction, et le diamètre minimal atteint pendant la journée. Cette contraction représente l'apport d'eau du phloème à la transpiration (Molz et Klepper, 1972; Jarvis, 1975);

- l'évolution nette du diamètre maximal journalier (DM), qui est la différence entre les diamètres maximaux mesurés avant le début de la contraction sur 2 journées consécutives. Une évolution nette positive du diamètre maximal (DM) correspond à la croissance journalière de la tige mesurée. En revanche, des valeurs négatives du $D M$ traduisent une réhumectation incomplète du phloème.

Pour déterminer des valeurs seuils correspondant à l'apparition de la contrainte hydrique, les auteurs s'appuient sur des valeurs de $\mathrm{CM}$ (Hu- guet, 1985), ou sur des valeurs de $C M$ et $D M$ (Garnier, 1985).

\section{Variation des diamètres des tiges en relation avec le potentiel de base}

Le potentiel de base (valeur particulière du potentiel hydrique foliaire mesurée avant le lever du soleil) peut être considéré (Meyer et Green, 1980; Katerji et Hallaire, 1984; Dwyer et Stewart, 1985) comme indicateur de l'équilibre sol-plante. Son évolution dans le temps reflète l'évolution de la réserve hydrique dans le sol (Dwyer et Stewart, 1985).

La figure 2a donne l'évolution de la variation des diamètres des tiges observées sur 7 plantes du 11 au 14 juin (jour $j-$ jour $j+3$ ). La figure $2 b$ donne les valeurs moyennes du potentiel de base et l'écart-type pendant les mêmes journées. Les valeurs moyennes journalières et l'écart-type de la contraction maximale (CM) et de l'évolution nette du diamètre maximal (DM) sont présentées dans le tableau I.

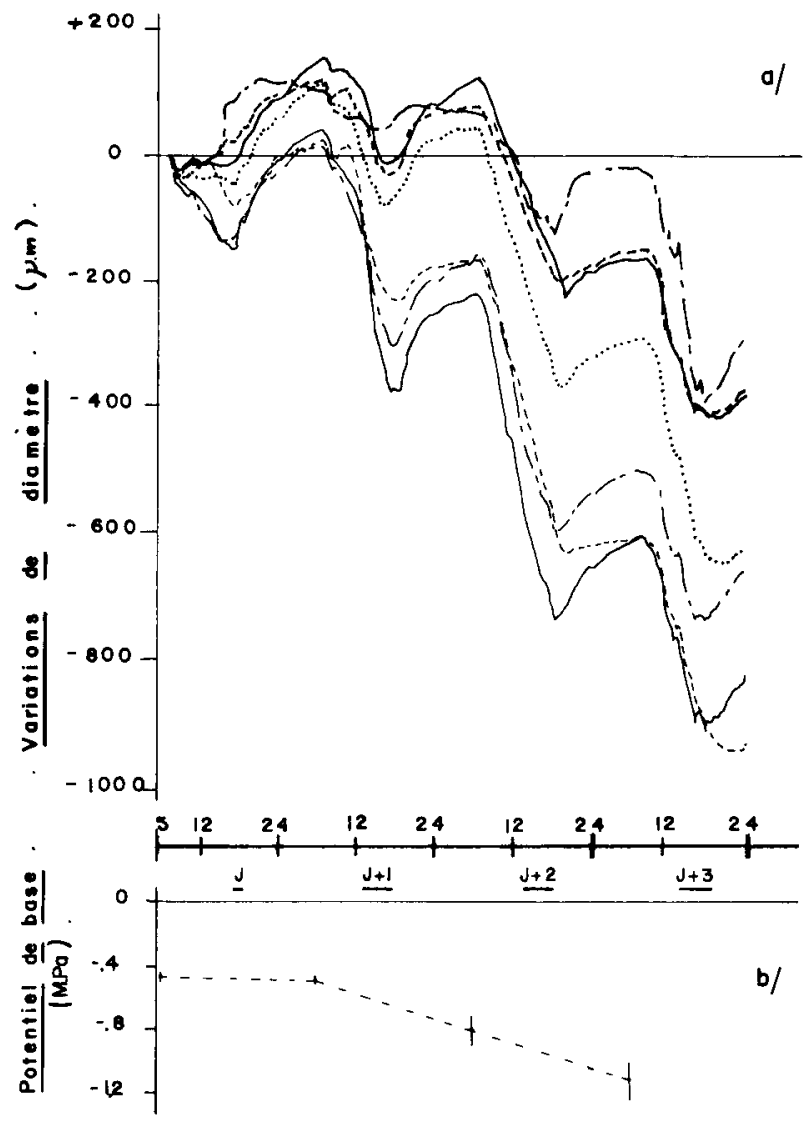

Fig 2. Evolution du jour $J$ à $J+3(11-14$ juin). a) de la variation du diamètre des tiges observée sur les 7 plantes étudiées; b) des valeurs moyennes du potentiel de base (les traits verticaux représentent l'écart type). 
Tableau 1. Valeurs moyennes journalières de l'écart type, de la contraction maximale $C M$ et de l'évolution nette du diamètre maximal $D M$ sur 7 plantes, pendant la période 11-14 juin $(\mathrm{J}-\mathrm{J}+3)$.

\begin{tabular}{lrrrr}
\hline & $J$ & $J+1$ & $J+2$ & $J+3$ \\
\hline \multirow{2}{*}{$\mathrm{DM}(\mu \mathrm{m})$} & 79 & -121 & -296 & $J+2$ \\
& \pm 50 & \pm 84 & \pm 103 & \\
$\mathrm{CM}(\mu \mathrm{m})$ & -76 & -215 & -356 & -308 \\
& \pm 50 & \pm 110 & \pm 112 & \pm 54 \\
\hline
\end{tabular}

On constate que les valeurs de potentiel de base sont pratiquement constantes au cours des 2 premières journées et restent voisines de $-0,4$ MPa, puis diminuent rapidement avec l'arrêt total de l'arrosage.

Au jour $\mathrm{J}$, les contractions $C M$ sont relativement faibles, avec ensuite des valeurs de $D M$ positives. La diminution de l'arrosage appliquée à partir du jour $\mathrm{J}+1$ conduit à des valeurs de $C M$ plus négatives et des valeurs de $D M$ plus basses par rapport à la journée précédente et ces phé- nomènes s'accentuent avec l'arrêt total de l'irrigation. Les valeurs seuils de $C M$ et $D M$ observées le jour $\mathrm{J}+2$, qui correspondent à la chute du potentiel de base, sont respectivement de $356 \mu \mathrm{m} \pm 112$ et de $-296 \mu \mathrm{m} \pm 103$ (tableau 1) . On note que la variabilité qui affecte ces valeurs moyennes journalières est importante, alors que les valeurs moyennes du potentiel de base sont au contraire beaucoup plus homogènes (fig $2 b$ ).

La figure $3 a$ donne l'évolution de la variation des diamètres des tiges observées sur 4 plantes des lots sec et irrigué (2 plantes par lot) du 16 au 23 septembre (jour $\mathrm{J}$ ' - jour $\mathrm{J}+6$ ). La figure $3 \mathrm{~b}$ donne les valeurs de potentiel de base observées sur les deux traitements sec et irrigué pendant les mêmes journées. Les valeurs de $D M$ et $C M$ observées sur les 4 capteurs retenus sont présentées dans le tableau II.

On constate que les valeurs de potentiel de base observées sur le lot irrigué sont relativement constantes et restent voisines de celles observées sur des plantes bien alimentées en eau au cours de l'expérience du mois de juin $\left(\psi_{b}=-0,35 \mathrm{MPa}\right)$. On note que les traitements sec et irrigué ont les mêmes valeurs de potentiel

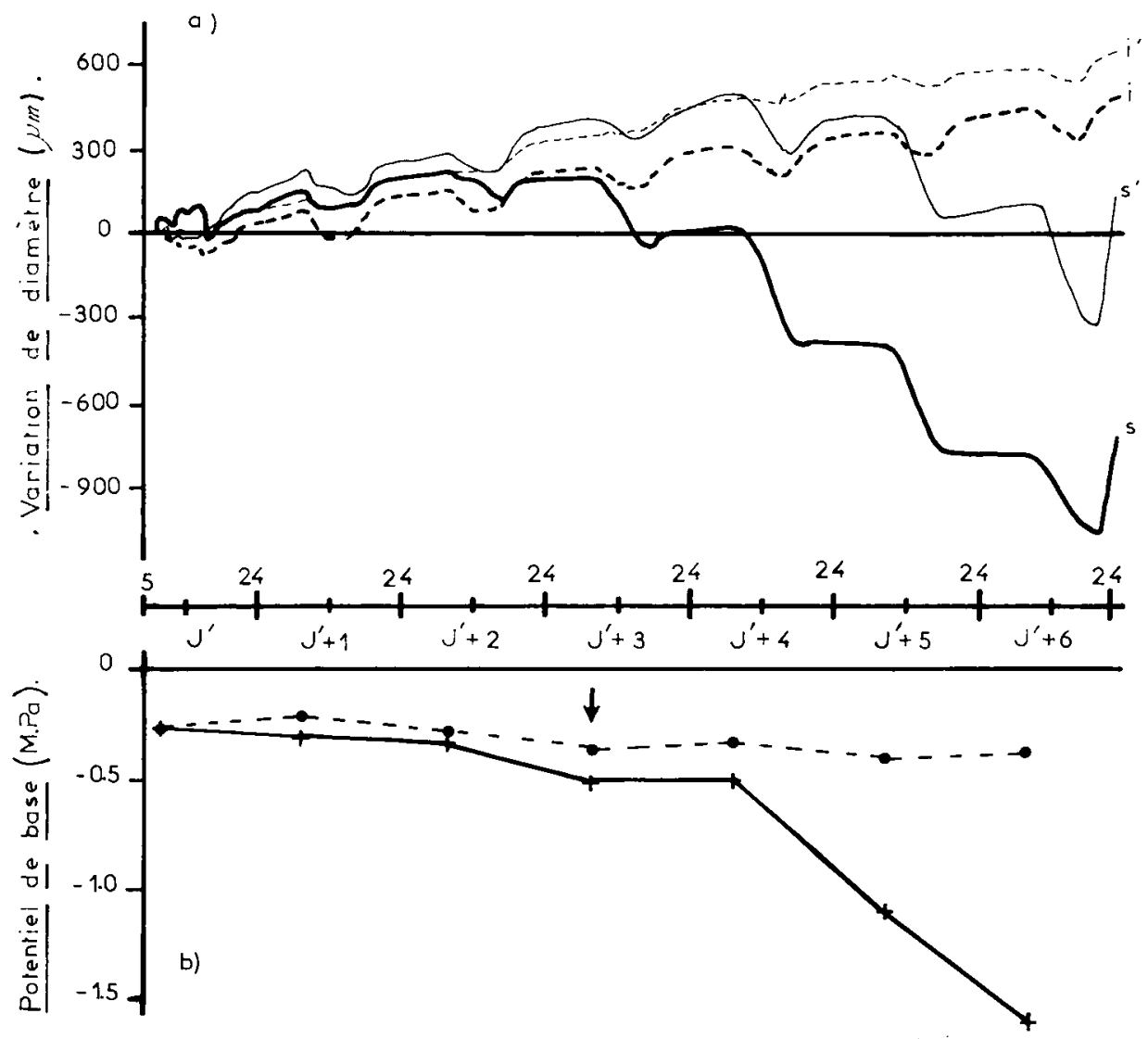

Fig 3. Évolution du jour J' à J'+ 6 (16 au 23 septembre) : a) de la variation du diamètre des tiges observée sur 2 plantes du lot irrigué (i et $i^{\prime}$ ) et 2 plantes du lot sec ( $s$ et $\left.s^{\prime}\right)$; b) des valeurs moyennes du potentiel de base observées sur les lots sec $(+-+)$ et irrigué $(0-0)$. La flèche indique le jour à partir duquel les valeurs horaires observées sur les 2 lots deviennent significativement différentes au seuil de $5 \%$. 
Tableau II. Valeurs journalières de la contraction maximale $C M$ et de l'évolution nette du diamètre maximal $D M$ sur 2 plantes du lot irrigué (i et $\left.i^{\prime}\right)$ et sur deux plantes du lot sec $\left(s\right.$ et $\left.s^{\prime}\right)$ pendant la période 16-23 septembre $\left(J^{\prime}-J^{\prime}+6\right)$.

\begin{tabular}{|c|c|c|c|c|c|c|c|c|c|}
\hline & & $J^{\prime}$ & $J^{\prime}+1$ & $J^{\prime}+2$ & $J^{\prime}+3$ & $J^{\prime}+4$ & $J^{\prime}+5$ & $J^{\prime}+6$ & Plante \\
\hline Lot irrigué & $\begin{array}{l}\mathrm{DM}(\mu \mathrm{m}) \\
\mathrm{CM}(\mu \mathrm{m})\end{array}$ & $\begin{array}{r}+85 \\
+110 \\
-80 \\
0\end{array}$ & $\begin{array}{r}+80 \\
+110 \\
-95 \\
-30\end{array}$ & $\begin{array}{l}+80 \\
+125 \\
-80 \\
-10\end{array}$ & $\begin{array}{r}+70 \\
+130 \\
-80 \\
-10\end{array}$ & $\begin{array}{l}+60 \\
+80 \\
-100 \\
-20\end{array}$ & $\begin{array}{l}+80 \\
+30 \\
-90 \\
-40\end{array}$ & $\begin{array}{l}-110 \\
-40\end{array}$ & $\begin{array}{l}i \\
i^{\prime} \\
i \\
i^{\prime}\end{array}$ \\
\hline Lot sec & $\begin{array}{l}\mathrm{DM}(\mu \mathrm{m}) \\
\mathrm{CM}(\mu \mathrm{m})\end{array}$ & $\begin{array}{r}+110 \\
+220 \\
0 \\
-20\end{array}$ & $\begin{array}{l}+60 \\
+60 \\
-60 \\
-90\end{array}$ & $\begin{array}{l}-20 \\
+130 \\
-90 \\
-60\end{array}$ & $\begin{array}{l}-180 \\
+90 \\
-240 \\
-70\end{array}$ & $\begin{array}{l}-400 \\
-80 \\
-400 \\
-210\end{array}$ & $\begin{array}{l}-380 \\
-310 \\
-380 \\
-360\end{array}$ & $\begin{array}{l}-260 \\
-410\end{array}$ & $\begin{array}{l}s \\
s^{\prime} \\
s \\
s^{\prime}\end{array}$ \\
\hline
\end{tabular}

de base jusqu'au jour $J^{\prime}+2$, puis des différences apparaissent au cours des journées suivantes, qui s'accentuent notamment à partir du jour $\mathrm{J}^{\prime}+$ 5.

La variation des diamètres des tiges observée sur les 2 plantes du traitement irrigué (désignées par $i$ et $i$ 'sur la figure 3a) présente une évolution classique : contraction au cours de la journée, avec ensuite une évolution nette positive du diamètre maximal. Les valeurs journalières $C M$ et $D M$ observées sur les plantes du lot irrigué sont hétérogènes, contrairement au cas du potentiel de base; en effet, ces valeurs évoluent entre -91 \pm 12 et $-21 \pm 16$ pour la $C M$ et entre $76 \pm 9$ et 98 \pm 37 pour la $D M$. Les 2 plantes de traitement $\mathrm{sec}$, (désignées par $s$ et $s^{\prime}$ sur la figure 3a) présentent la même évolution jusqu'au jour $\mathrm{J}^{\prime}+2$, puis des différences apparaissent à partir du jour $\mathrm{J}^{\prime}+3$ (cas de la plante s) ou $24 \mathrm{~h}$ plus tard (cas de la plante $s^{\prime}$ ). Dans les 2 cas, ces différences se traduisent par un accroissement de la contraction maximale, avec ensuite une évolution négative nette du diamètre maximal, et ce phénomène s'accentue très fortement au cours des journées suivantes. On constate enfin que les valeurs de variation de diamètre observées sur les 2 plantes du lot sec, qui correspondent à la chute du potentiel de base, sont hétérogènes : -240 et $-70 \mu \mathrm{m}$ pour la $C M$ et -180 et $+90 \mu \mathrm{m}$ pour la $D M$.

\section{Variation des diamètres des tiges et fonctionnement hydrique et photosynthétique des plantes}

Sur la figure 4 sont présentées les valeurs horaires moyennes du potentiel hydrique foliaire, de la résistance stomatique moyenne, de l'assimilation nette et de la transpiration, ainsi que les variations des diamètres des tiges extrêmes (maximales et minimales) relevées sur les 7 plantes étudiées pendant les 4 journées de la $1^{\text {re }}$ expérience (11-14 juin). Notons que le potentiel hydrique foliaire est un indicateur direct de l'état hydrique de la plante, tandis que les échanges gazeux (résistance stomatique, photosynthèse, transpiration) sont plutôt des indicateurs indirects

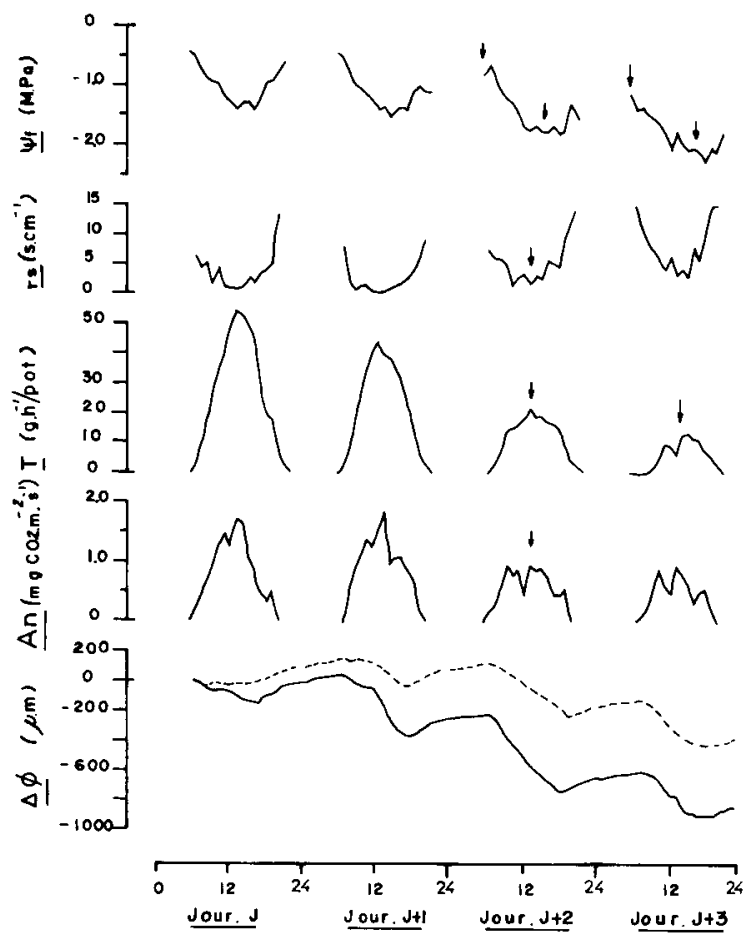

Fig 4. Évolution du jour $\mathrm{J}$ à jour $\mathrm{J}+3$ (du 11-14 juin) des valeurs horaires du potentiel hydrique foliaire $\psi_{f}$, de la résistance stomatique $r s$, de la transpiration $T$, de l'assimilation nette $A n$ et des variations du diamètre des tiges extrêmes (maximales et minimales) $\Delta \sigma$, observées sur les 7 plantes étudiées. La flèche indique l'heure à partir de laquelle les valeurs horaires deviennent significativement différentes de la journée précédente, au seuil de $5 \%$. 
puisqu'il s'agit de fonctions susceptibles d'être modifiées par le changement de l'état hydrique de laplante.

Les données du potentiel hydrique foliaire et de la résistance stomatique subissent au cours de la journée une évolution tout à fait classique : une diminution régulière à partir de $5 \mathrm{~h}$ du matin, atteignant vers $13-15 \mathrm{~h}$ les valeurs minimales, puis une reprise par la suite lorsque le rayonnement solaire diminue. Celles de la transpiration et de la photosynthèse présentent une évolution inverse : les valeurs minimales sont observées en début et en fin de journée, tandis que les valeurs maximales sont atteintes en milieu de journée. On note également que les paramètres précédents évoluent d'une façon identique au cours des journées $\mathrm{J}$ et $\mathrm{J}+1$, sauf pour la transpiration qui présente une diminution au cours de la journée $J+1$. II est très difficile d'attribuer à cette différence une signification biologique, compte tenu des fuites observées, particulièrement ce jour-là, à la suite de l'arrosage des pots pesés.

À partir $\mathrm{du}$ jour $\mathrm{J}+2$, on constate au contraire que les valeurs de potentiel de base, les valeurs horaires minimales du potentiel hydrique foliaire et de résistance stomatique, ainsi que les valeurs horaires maximales de l'assimilation nette et de la transpiration, deviennent significativement différentes par rapport aux journées précédentes. Les valeurs seuils de $C M$ et $D M$ correspondant aux modifications observées sur les indicateurs de l'état hydrique de la plante sont identiques à celles déterminées pour le changement significatif du potentiel de base $(-356 \pm 112$ pour la $C M$ et $-296 \pm 103$ pour la $D M$ ).

Sur la figure 5 sont présentées les valeurs horaires moyennes du potentiel hydrique foliaire et de la résistance stomatique de la face supérieure des feuilles observées sur les lots sec et irrigué pendant 5 journées consécutives du 17 au 23

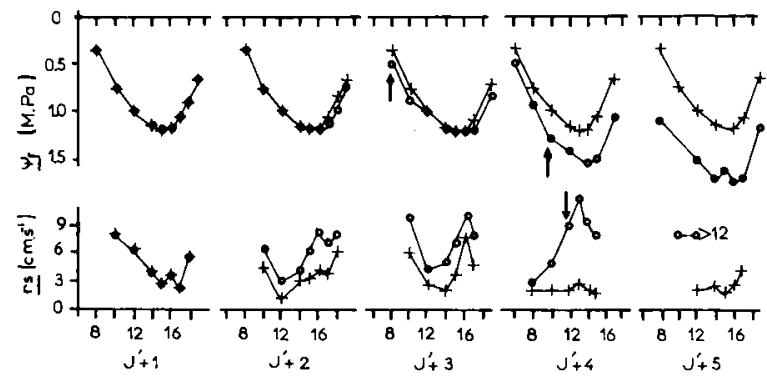

Fig 5. Evalution du jour J'+1 au jour $J^{\prime}+5$ (17-22 septembre) des valeurs horaires du potentiel hydrique foliaire $\psi_{f}$ et de la résistance stomatique de la face supérieure des feuilles rs observées sur les lots $\sec (0-0)$ et irrigué $(\longrightarrow)$. La flèche indique l'heure à partir de laquelle les valeurs horaires observées sur les 2 lots deviennent significativement différentes au seuil de $5 \%$. septembre (jours $\mathrm{J}^{\prime}+1$ au $\mathrm{J}^{\prime}+5$ ). Les données relatives à la journée du 16 septembre (jour J') ne sont pas présentées sur la figure 5 , dans la mesure où elles sont identiques à celles observées le jour $\mathrm{J}+1$.

La cinétique journalière du potentiel hydrique foliaire observée du jour $J^{\prime}+1$ au $J^{\prime}+5$ sur le lot irrigué est extrêmement stable. On note également que les traitements sec et irrigué ont les mêmes valeurs du potentiel hydrique foliaire pendant les 2 premières journées. À partir du jour $\mathrm{J}^{\prime}+3$, le potentiel de base est significativement plus bas, mais aux heures chaudes de la journée, les potentiels hydriques ont les mêmes valeurs. Dès le jour J' +4 , les potentiels hydriques sont significativement différents à toutes les heures de la journée, et cette différence s'accentue la journée suivante. En raison de la rosée sur les feuilles en début de journée, il n'est pas possible de mesurer la résistance stomatique avant $10 \mathrm{~h}$. Jusqu'au jour $\mathrm{J}$ ' +1 , les 2 lots ont des valeurs de résistance stomatique identiques. Des différences apparaissent lors des journées suivantes, mais ne deviennent significatives qu'à partir du jour $J^{\prime}+4$, c'est-à-dire au moment où les potentiels hydriques foliaires sont systématiquement différents entre les plantes des 2 traitements. Les valeurs de $C M$ et $D M$ correspondant à ces différences observées de la résistance stomatique et du potentiel hydrique foliaire sont respectivement de -210 et $400 \mu \mathrm{m}$ pour la $\mathrm{CM}$ et de $-400 \mu \mathrm{m}$ et $-80 \mu \mathrm{m}$ pour la $D M$.

\section{DISCUSSION ET CONCLUSION}

L'ensemble des résultats obtenus dans le cadre de notre étude fournit des éléments de réponse aux 3 questions posées au départ.

\section{Indicateur de l'état hydrique de la plante à retenir}

Nous avons vérifié d'abord que la variation du diamètre de la tige agissait en synchronisation avec tous les indicateurs de l'état hydrique de la plante retenus dans le cadre de cette étude. Nous préconisons toutefois de retenir le potentiel de base pour déterminer les valeurs seuils de variation de diamètre, de préférence au potentiel foliaire minimal mesuré au début de l'après-midi et proposé par Garnier, 1985. Deux raisons dictent ce choix : 
- le potentiel de base apparaît dans certains cas (expérience du mois de septembre) plus sensible que les autres indicateurs au changement de régime d'alimentation hydrique;

- le potentiel hydrique foliaire minimal n'est pas un indicateur sensible chez les espèces à comportement isohydrique (comportement qui se traduit par le maintien d'un potentiel hydrique élevé dans les tissus végétaux pendant la période chaude de la journée), comme la tomate par exemple. II l'est en revanche chez les espèces à comportement hydrique anisohydrique (comportement inverse au cas précédent), comme l'aubergine. Le potentiel de base, quant à lui, présente l'avantage d'être déterminé à une heure de la journée où le comportement isohydrique ou anisohydrique de la culture ne se manifeste pas encore. Une discussion détaillée sur ce point se trouve dans Katerji et al, 1988).

\section{Détermination des valeurs seuils}

La détermination des valeurs seuils de variation de diamètre qui permettent de diagnostiquer l'apparition de la contrainte hydrique peut se faire de façon qualitative et quantitative. Nous avons noté, en effet, que les modifications du fonctionnement hydrique et photosynthétique, à la suite d'un changement dans l'alimentation hydrique de la plante, coïncident systématiquement avec l'apparition d'une évolution négative nette du diamètre maximal, quel que soit le nombre de plantes étudié dans l'échantillon. L'apparition d'une valeur négative de $D M$ peut être donc considérée comme un critère qualitatif pour diagnostiquer l'apparition de la contrainte hydrique. On peut se demander, à la suite de ces observations et sous réserve d'autres vérifications réalisées au champ sur d'autres espèces végétales, s'il ne serait pas possible de diagnostiquer une contrainte hydrique par le seul suivi des variations de diamètre.

\section{Précision des valeurs seuils}

La détermination quantitative des valeurs seuils de variation du diamètre peut se faire à partir de 2 critères : la contraction maximale $C M$ et l'évolution nette de diamètre maximal $D M$. Le coefficient de variation affectant les valeurs seuils de $D M$ et $C M$ déterminées à partir de 7 capteurs est de l'ordre de $31 \%$ pour la $C M$ et de $34 \%$ pour la $D M$. Cette variabilité est supérieure à celle observée sur certains indicateurs de l'état hydrique de la plante, comme le potentiel de base ou le potentiel hydrique foliaire (Katerji, 1990), mais elle se rapproche de la variabilité observée sur d'autres indicateurs tels que la résistance stomatique (Katerji, 1990) ou la température radiative (Katerji et al, 1988). Nous n'avons pas trouvé chez Huguet (1985) ni Garnier (1985) de renseignements concernant la précision des valeurs seuils de $C M$ et $D M$ qu'ils déterminent. En revanche, l'étude réalisée par Benoit (1985) sur le maïs fournit des éléments de comparaison : l'auteur a noté, en effet, que les valeurs moyennes de $C M$ déterminées à partir de 6 capteurs sur des plantes de maïs soumises à une contrainte hydrique sont affectées d'un coefficient de variation allant de 30 à $40 \%$, ce qui est tout à fait comparable aux résultats obtenus dans cette étude. Les bioprogrammateurs mis en service depuis 1988 et dotés d'un nombre de capteurs plus important devraient permettre une meilleure précision des valeurs seuils de $D M$ et $C M$.

La programmation de l'irrigation repose sur le diagnostic de l'apparition de la contrainte hydrique, mais d'autres éléments sont indispensables pour justifier une irrigation pertinente, par exemple la sensibilité du stade phénologique (Robelin, 1967) ou de l'espèce (Puech et al, 1976) à un manque d'eau. Huguet et al, 1987, proposent comme seuil critique la valeur moyenne de $C M$ observée le jour où l'évolution nette de diamètre manifeste une rupture nette de tendance. À titre d'exemple, dans l'expérience du mois de juin (figs 2 et 4 ), ce phénomène se produit le jour $\mathrm{J}+1$ et le seuil critique de $C M$ sera alors $(-220 \mu \mathrm{m})$. On note que la détermination du seuil critique, selon la proposition de Huguet et al (1987) revient à anticiper par l'irrigation la modification du fonctionnement de la plante. À notre avis, si ce choix se justifie dans le cas d'une contrainte hydrique affectant la plante pendant un stade particulièrement sensible, il peut provoquer dans les autres situations, comme nous l'avons constaté dans une étude précédente (Katerji, 1977), une croissance végétative abondante et un allongement du cycle de la culture, sans pour autant provoquer une augmentation notable du rendement. II serait donc prudent d'envisager des seuils critiques susceptibles d'être modifiés selon la sensibilité du stade phénologique et de l'espèce végétale à la contrainte hydrique. 


\section{RÉFÉRENCES}

Benoit V (1985) Essai de mise au point de la conduite d'irrigation du maïs par mesure micrométrique. Mémoire ENSFA Rennes

Brough DW, Jones HG, Grace J (1986) Diurnal changes in water content of the stems of apple trees, as influenced by irrigation. Plant Cell Environ $9,1-7$

Daunay MC, Malet P, Schoch PG (1986) Facteurs agroclimatiques influençant la résistance stomatique (Solanum melongena). Agronomie 6, 615-622

Dwyer LM, Stewart DW (1985) Indicators of water stress in corn (Zea mays L). Can J Plant Sci 64, 537-546

Garnier E (1985) Dessèchement du sol et indicateurs physiologiques de l'état hydrique chez le pêcher : application au déclenchement de l'irrigation. Thèse docteur ingénieur, ENSA Montpellier

Huguet JG (1985) Appréciation de l'état hydrique d'une plante à partir des variations micrométriques de la dimension des fruits ou des tiges en cours de journées. Agronomie 5, 733-741

Huguet JC, Benoit V, Orlando P (1987) Application de la micromorphométrie sur tige au pilotage de l'irrigation du maïs. In: Le maïs et l'eau. Vol 1, colloque alimentation hydrique du maïs, AGPM, ITCF, 8-9 décembre 1987, $16 p$

Jarvis PG (1975) Water transfer in plants. In : Heat and mass transfer in the biosphere (de Vries DA, Afgan NH eds) John Wiley \& Sons, New York, 369394

Katerji N (1977) Contribution à l'étude de l'évapotranspiration réelle du blé tendre d'hiver. Application à la résistance du couvert en relation avec certains facteurs du milieu. Thèse docteur ingénieur Université de Paris VII

Katerji N (1990) Exploration et analyse de l'hétérogénéité spatiale au champ de deux indicateurs de l'état hydrique de la plante : la résistance stomatique et le potentiel hydrique foliaire. In : L'expérimentation agricole et l'alimentation en eau des plantes. Colloque Versailles, AFMEX, 25-26 janvier $1989,77-90$
Katerji N, Hallaire M (1984) Les grandeurs de références utilisables dans l'étude de l'alimentation en eau des cultures. Agronomie 4, 999-1008

Katerji N, Itier B, Fereira I (1988) Étude de quelques critères indicateurs de l'état hydrique d'une culture de tomate en région semi-aride. Agronomie 8, 425433

Klepper B, Browning VD, Taylor HM (1971) Stem diameter in relation to plant water status. Plant Physiol $48,683-685$

Kozlowski TT (1967) Diurnal variations in stem diameters of small trees. Bot Gaz 123, 60-68

Meyer WS, Green GC (1980) Water use by wheat and plant indicators of available soil water. Agron $J$ $72,253-257$

Molz FJ, Klepper B (1972) Radial propagation of water potential in stems. Agron J 64, 469-473

Pereira JS, Kozlowski TT (1976) Diurnal and seasonal changes in water balance of Abies balsamea and Pinus resinosa. Oecol Plant 11, 397-412

Powell DBB, Thorpe MR (1977) Dynamic aspects of plant water relations. In : Environmental effects on crop physiology. (JJ Landesberg, CV Cutting, eds). Academic Press, London, 259-279

Puech J, Marty JR, Maertens C (1976) Efficience de l'eau consommée par divers végétaux et application à la valorisation de l'irrigation. Bull Tech Inf 306, 41-53

Robelin M (1967) Action et arrière action de la sécheresse sur la croissance et la production du tournesol. Ann Agron 6, 579-599

Scholander PF, Hammel HT, Bradsteet ED, Hemmingsen EA (1965) Sap pressure in vascular plants. Science 148, 339-346

Schoch PG, Katerii N, Rimgoto $P$, Tchamitchian $M$, Malet $P$, L'Hotel JC, Daunay MC (1987) Influence du niveau d'alimentation hydrique sur les variations du diamètre des tiges, du potentiel hydrique, de la résistance stomatique, de la transpiration et de la photosynthèse de l'aubergine (Solanum melongena L). Agric For Meteorol 40, 89-104

So MB, Reicosky DC, Taylor HM (1979) Utility of stem diameter changes as predictors of plant canopy water potential. Agron J71, 707-713 